\title{
Malignant carotid body tumor presenting with myelopathy: case report
}

\author{
Dhruve S. Jeevan, MD, Mohamed Saleh, MD, Michael LaBagnara, MD, Jayson A. Neil, MD, and \\ Virany H. Hillard, MD
}

Department of Neurosurgery, New York Medical College, Valhalla, New York

Malignant carotid body tumors are rare, with spread of the tumor mostly noted in regional lymph nodes. Vertebral metastases are an exceedingly rare presentation, only reported in isolated case reports, and present a diagnostic and management challenge. A case of widespread vertebral metastasis, presenting with myelopathy, from a carotid body tumor is discussed in this paper, along with management strategies.

http://thejns.org/doi/abs/10.3171/2015.8.SPINE13483

KEY WORDS carotid body tumor; paraganglioma; vertebral metastasis; oncology

$\mathrm{P}$ ARAGANGLIOMAS (PGs) are rare extraadrenal neuroendocrine tumors that may appear in all ages, with the highest incidence in the fifth decade of life. PGs are often classified based on their site of origin, with $85 \%$ found in the abdomen, $12 \%$ in the chest, and $3 \%$ in the head and neck region. Carotid PGs (or carotid body tumors) are the most common of the head and neck tumors. Approximately $75 \%$ of all PGs do not produce catecholamines, and as a result present primarily as incidental findings or through mass effect, leading to a late diagnosis. ${ }^{16}$ Most PGs are benign, with only 4\%-15\% developing metastases, ${ }^{11,12}$ usually isolated to regional lymph nodes. While surgery is commonly curative in benign PGs, the prognosis for malignant PGs remains poor, with 5-year mortality rates greater than $50 \% .^{20}$ In this paper we present a rare case of a malignant, nonsecreting, carotid body PG with spinal metastasis, and discuss adjuvant treatments and management strategies.

\section{Case Report}

History and Examination

A 77-year-old woman presented to our institution with a 5-week history of progressive weakness of her lower extremities. She had experienced an increasing frequency of falls, to the point that the patient had become nonambulatory over the last 2 days. No bowl or bladder disturbances had been noted, but a loss of sensation to the bilateral lower extremities was also described. No other constitutional symptoms were described, including absence of weight loss, heat/cold intolerance, episodes of diaphoresis, or palpitations. Examination of the patient revealed bilateral lower-extremity motor weakness in all muscle groups, with loss of light touch and pinprick sensation. A sensory level was determined at the costal margin, with normal rectal tone. Upper extremity strength was normal. Systemic examination revealed a small pulsatile mass in the right neck medial to the sternocleidomastoid. The mass was firm with free mobility in the rostrocaudal direction without medial lateral mobility. There were no other palpable masses or lymphadenopathy, and normal thyroid palpation. No other systemic findings were noted.

An initial CT scan performed on the vertebral column revealed extensive spinal metastatic disease (Fig. 1A), with the largest destructive lesion found at T-3, demonstrating extensive erosion of the vertebral body and pedicles. Epidural extension of the lesion was found at this level with associated spinal stenosis and cord compression. CT of the neck, along with chest, abdomen, and pelvic imaging revealed a $2 \times 3 \times 3-\mathrm{cm}$ enhancing lesion centered on the right common carotid artery bifurcation (Fig. 2). No other organ lesions were found, including normal thyroid imaging. At this time, imaging was suspicious of a carotid body tumor, although likely as an incidental finding, as well as extensive metastatic disease of an unknown primary site.

ABBREVIATIONS $\quad$ |131-MIBG = iodine-131 meta-iodobenzylguanidine; $P G=$ paraganglioma.

SUBMITTED May 28, 2013. ACCEPTED August 11, 2015.

INCLUDE WHEN CITING Published online January 1, 2016; DOI: 10.3171/2015.8.SPINE13483. 

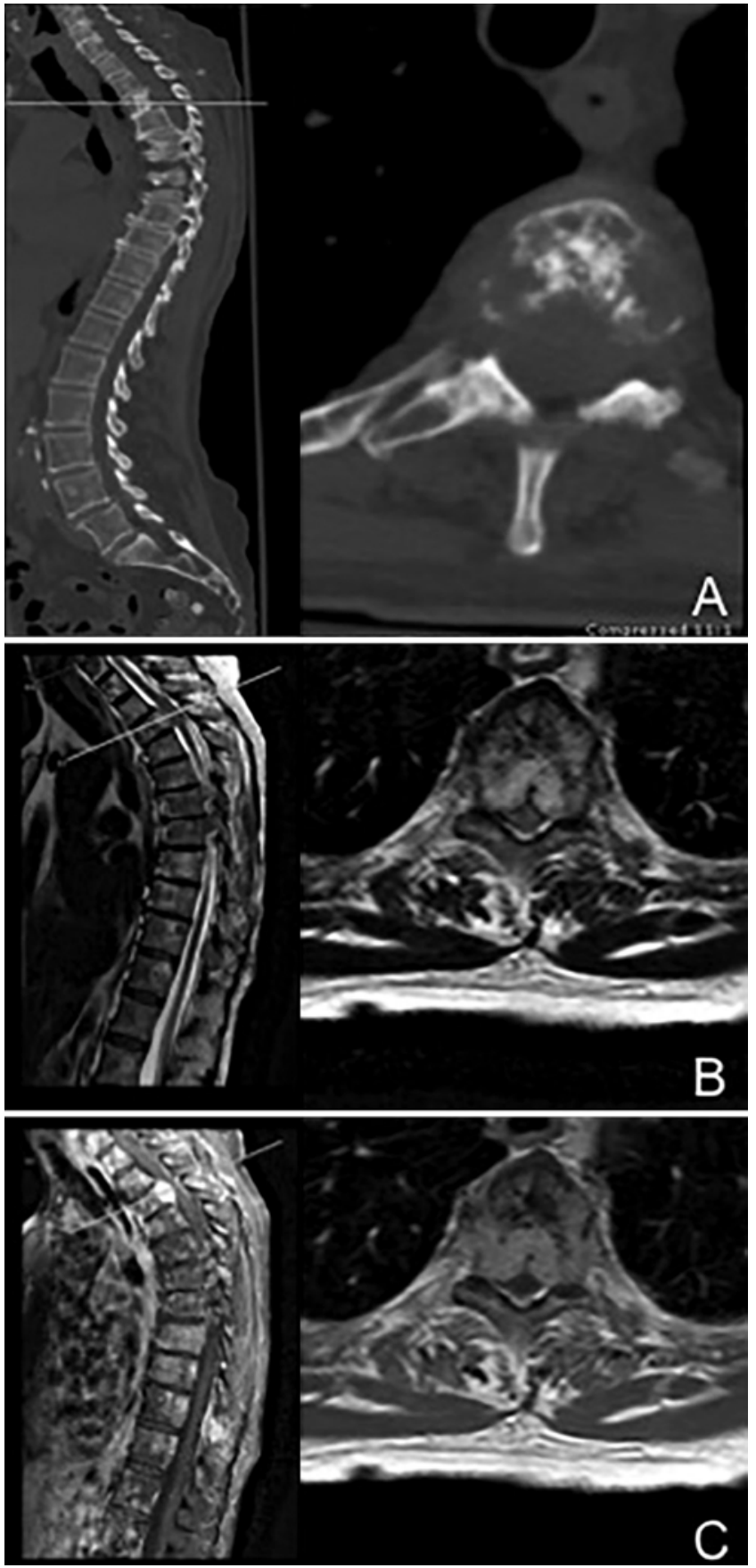

FIG. 1. Sagittal (left) and axial (right) thoracic CT (A) and MRI (B and C) showing a lytic lesion of the T-3 vertebral body, with extension into the epidural space occupying the spinal canal and causing severe spinal cord compression. Multiple other lytic lesions are identified throughout the thoracolumbar spine.

\section{Operation}

As a result of these findings further investigation was planned for the patient, including mammography, urine and serum metanephrines, serum calcitonin levels, and MRI. Serum laboratory values revealed no abnormalities, and the patient demonstrated absence of urinary metanephrines. MRI confirmed extensive osseous metastases, most advanced at T-3, where extradural extension was re- sulting in cord compression with associated signal intensity changes (Fig. 1B and C). The right carotid body lesion was again visualized, with splaying of the internal and external carotid arteries, confirming a suspected diagnosis of a carotid body PG (Fig. 2). Due to the rarity of malignant degeneration of carotid body PGs presenting with vertebral metastases, the patient underwent radiologically guided needle biopsy of her right carotid body lesion, as well as an L-3 vertebral body lesion biopsy. Although the pathology from the vertebral biopsy was inconclusive, histological examination of the right carotid body lesion confirmed the suspicion of a PG. Due to progressive neurological decline and imaging confirmation of spinal compression, the patient underwent an operation for thoracic decompression with posterolateral fixation from T-1 to T-5. A wide laminectomy was performed between T-2 and T- 4 with partial bilateral facetectomy at T-3. At this time, a highly vascular epidural mass was encountered from which a biopsy specimen was obtained. Resection of this epidural mass was attempted, but due to the high vascularity of the lesion, extensive resection was abandoned once the spinal canal was believed to be adequately decompressed. Histological examination of this specimen was consistent with a PG, with visualized tumor cells arranged in distinct nests separated by fibrovascular stroma and sustenacular cells (Fig. 3). On immunohistochemical examination, the chief cells located within the nests were positive for chromogranin, while sustanacular cells stained positively for S100 protein. Two independent pathologists, following outside consultation, confirmed the pathology.

\section{Postoperative Course}

Due to the rarity of this disease there are no current guidelines on management. Considering the patient's age and neurological status, she received local radiation therapy of 40 Gy in 20 fractions to the local disease at T-3, alongside sunitinib chemotherapy. She remains on sunitinib treatment 2 years after surgical therapy with stable disease, with no new lesions. MRI performed 2 years postoperatively demonstrated a significant reduction of epidural disease at T-3, with no evidence of cord compression. The suspected primary lesion at the carotid bifurcation also remains stable in size. Neurologically, the patient has made a complete recovery with independent ambulation.

\section{Discussion}

Malignant carotid PGs are defined by the pathological confirmation of tumor cells at distant locations rather than histological grading of the primary tumor. ${ }^{17}$ However, the incidence of metastasis is rare, representing only $4 \%$ to $15 \%$ of all head and neck tumors, $, 311,12$ with only a $6 \%$ reported rate for carotid body tumors. ${ }^{8}$ The majority of these metastases occur in regional lymph nodes (50\%), with the remainder occurring in the liver and lungs. Vertebral metastases are exceedingly rare, with only isolated case reports describing them. ${ }^{4,5,18,21}$ Our paper reports the first case in the literature in which a patient primarily presented with widespread vertebral metastasis with spinal compression from a carotid body PG. 

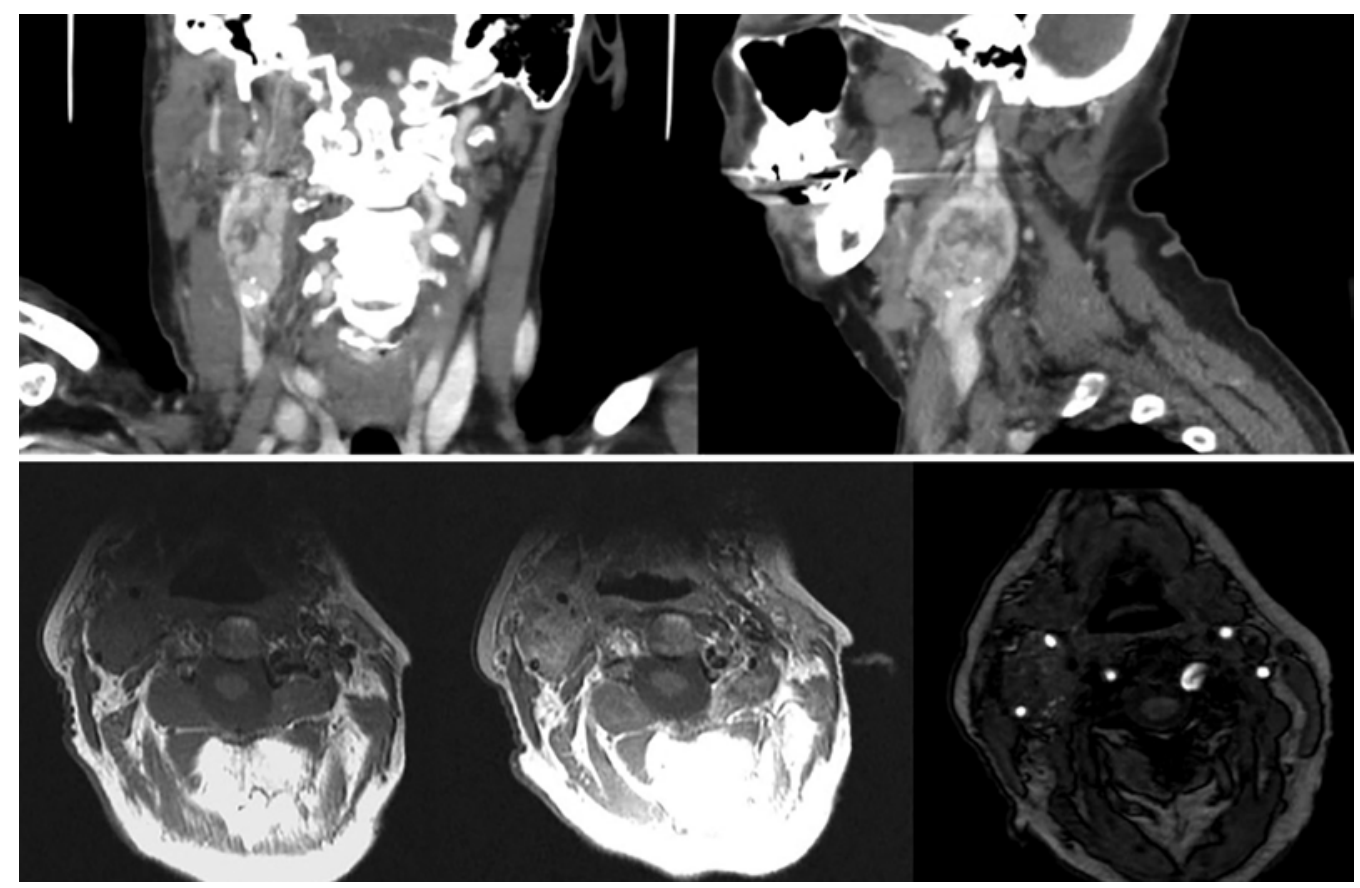

FIG. 2. CT scans (upper row) and MR angiograms (lower row) of the neck demonstrate an enhancing right carotid body mass consistent with a carotid body PG, with splaying of the internal and external carotid branches.

In those patients with regionally metastatic disease, primary resection with adjuvant radiation therapy offers a prognostic advantage. However, due to the rarity of distant organ metastases, optimal treatment strategies remain to be identified. These cases are often treated with systemic chemotherapeutics, but this strategy arises from studies that have focused on thoracic and abdominal PGs. These tumors remain biologically distinct compared with head and neck PGs. ${ }^{9}$

PGs can be classified as sympathetic when arising from the sympathetic autonomic nervous system of the thorax, abdomen, or pelvis, or parasympathetic when arising in the paraganglia of the head and neck. Sympathetic tumors are of chromaffin origin and are often secretory, resulting in palpitations, headaches, diaphoresis, and weight loss due to the effects of systemic catecholamines. ${ }^{3,17}$ A significant number of sympathetic PGs (15\%-23\%) are meta-

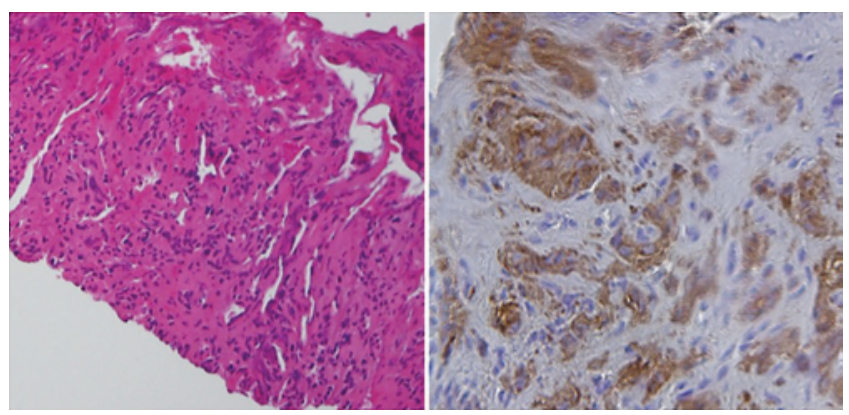

FIG. 3. High-magnification histopathological specimens of the thoracic lesion demonstrating nests of chromogranin-positive cells in a sustenacular cell background. These are typical findings of a PG. H \& E (left) and chromogranin (right). Original magnification $\times 10$ (left) and $\times 100$ (right). Figure is available in color online only. static. In contrast, head and neck parasympathetic PGs are hormonally silent and rarely malignant. As a result, they often present incidentally or through local mass effect. ${ }^{16}$ Presentation with distant metastases is extremely rare and is often only reported as a recurrence several years following primary tumor resection.

Metastatic disease to the vertebrae is determined by radiological imaging (CT and MRI), although neither of these modalities aid in differentiating tumor types. Thus, diagnosis is only confirmed by histological and immunohistochemical analysis of tissue. There have been several reported instances in which metastatic medullary carcinoma of the thyroid was believed to represent a PG.? The pathological similarity of these lesions is well established, ${ }^{8,22}$ although PGs usually do not stain for calcitonin, further emphasizing the importance of thyroid scans and serum calcitonin levels in routine evaluation of suspected metastatic PGs. Radionucleotide studies can be used to determine metastatic disease in some patients. I $^{131}$ MIBG (iodine-131 meta-iodobenzylguanidine) has shown increased uptake by chromaffin cells, even in nonsecreting tumors. This imaging modality was considered in our own patient due to the nondiagnostic nature of 2 needle biopsies. However, the need for spinal decompression for worsening neurological status led us to proceed directly to surgery. In patients where the diagnosis can been made preoperatively, angiographic embolization of major feeding blood vessels can be performed to reduce intraoperative blood loss during resection. Considering the extent of metastatic disease present in our patient, it was not believed that the morbidity of complete resection would offer any survival advantage, and hence angiography was not considered.

The rarity of metastatic carotid body tumors makes 
their management clinically challenging. External beam radiation therapy remains palliative in the treatment of spinal metastasis, with response more effective in younger patients. ${ }^{13}$ Limited metastasis to isolated vertebrae may benefit from resection following embolization, and adds to the effectiveness of radiation therapy. ${ }^{14} \mathrm{I}^{131}$-MIBG has recently been shown to be of therapeutic benefit. Where uptake is noted, this radio-iodinated treatment can produce palliation of bone pain, ${ }^{10}$ and has been observed to show a partial tumor response with a 75\% 5-year survival rate..$^{2,6,19}$ Chemotherapy remains the mainstay of treatment for systemic metastatic tumors. Various agents have been used, with combinations including cisplatin and vincristine. Although no consistent benefit has been demonstrated, up to $92 \%$ can achieve stable disease. ${ }^{13,15}$ Due to the small number of cases and the differences in groups receiving various treatment modalities, direct comparisons are difficult, and no proven treatment guidelines exist. Sunitinib, a potent inhibitor of multiple tyrosine kinase receptors, and an effective antiangiogenic drug, has been reported in some case series to benefit patients with sympathetic PG, but its role in head and neck PGs remains to be elucidated.

\section{Conclusions}

A case of vertebral metastasis from a malignant carotid body tumor is presented. This case highlights the need for clinical suspicion of this rare entity and the difficulties faced in its management. Resection with radiation therapy remains the mainstay of treatment where feasible, and systemic chemotherapy aids in systemic control.

\section{References}

1. Ayala-Ramirez M, Chougnet CN, Habra MA, Palmer JL, Leboulleux S, Cabanillas ME, et al: Treatment with sunitinib for patients with progressive metastatic pheochromocytomas and sympathetic paragangliomas. J Clin Endocrinol Metab 97:4040-4050, 2012

2. Bomanji JB, Hyder SW, Gaze MN, Gacinovic S, Costa DC, Coulter C, et al: Functional imaging as an aid to decisionmaking in metastatic paraganglioma. Br J Radiol 74:266269, 2001

3. Branham GH, Gnepp DR, O'McMenomey S, Friedman WH: Malignant paraganglioma-a case report and literature review. Otolaryngol Head Neck Surg 101:99-103, 1989

4. Brown JW, Burton RC, Dahlin DC: Chemodectoma with skeletal metastasis: report of two cases. Mayo Clin Proc 42:551-555, 1967

5. Coulson WF: A metastasizing carotid-body tumor. J Bone Joint Surg Am 52:355-360, 1970

6. Fitzgerald PA, Goldsby RE, Huberty JP, Price DC, Hawkins RA, Veatch JJ, et al: Malignant pheochromocytomas and paragangliomas: a phase II study of therapy with high-dose ${ }^{131}$ I-metaiodobenzylguanidine ( $\left.{ }^{131} \mathrm{I}-\mathrm{MIBG}\right)$. Ann N Y Acad Sci 1073:465-490, 2006

7. Gongal DN, Mack GJ, Vaidya BB: Extradural metastasis from a presumed carotid-body tumour producing paraplegia: a case report. Can J Surg 14:410-412, 1971
8. Harrington SW, Clagett OT, Dockerty MB: Tumors of the carotid body: clinical and pathologic considerations of twenty tumors affecting nineteen patients (one bilateral). Ann Surg 114:820-833, 1941

9. Joynt KE, Moslehi JJ, Baughman KL: Paragangliomas: etiology, presentation, and management. Cardiol Rev 17:159164,2009

10. Khafagi F, Egerton-Vernon J, van Doorn T, Foster W, McPhee IB, Allison RW: Localization and treatment of familial malignant nonfunctional paraganglioma with iodine-131 MIBG: report of two cases. J Nucl Med 28:528531,1987

11. Kupferman ME, Hanna EY: Paragangliomas of the head and neck. Curr Oncol Rep 10:156-161, 2008

12. Lee JH, Barich F, Karnell LH, Robinson RA, Zhen WK, Gantz BJ, et al: National Cancer Data Base report on malignant paragangliomas of the head and neck. Cancer 94:730737,2002

13. Moskovic DJ, Smolarz JR, Stanley D, Jimenez C, Williams MD, Hanna EY, et al: Malignant head and neck paragangliomas: is there an optimal treatment strategy? Head Neck Oncol 2:23, 2010

14. North CA, Zinreich ES, Christensen WN, North RB: Multiple spinal metastases from paraganglioma. Cancer 66:22242228, 1990

15. Patel SR, Winchester DJ, Benjamin RS: A 15-year experience with chemotherapy of patients with paraganglioma. Cancer 76:1476-1480, 1995

16. Plouin PF, Gimenez-Roqueplo AP: Pheochromocytomas and secreting paragangliomas. Orphanet J Rare Dis 1:49, 2006

17. Romanski R: Chemodectoma (non-chromaffinic paraganglioma) of the carotid body with distant metastases; with illustrative case. Am J Pathol 30:1-13, 1954

18. Say CC, Hori J, Spratt J Jr: Chemodectoma with distant metastasis: case report and review of the literature. Am Surg 39:333-341, 1973

19. Sisson JC, Frager MS, Valk TW, Gross MD, Swanson DP, Wieland DM, et al: Scintigraphic localization of pheochromocytoma. N Engl J Med 305:12-17, 1981

20. Welander J, Söderkvist P, Gimm O: Genetics and clinical characteristics of hereditary pheochromocytomas and paragangliomas. Endocr Relat Cancer 18:R253-R276, 2011

21. Whimster WF, Masson AF: Malignant carotid body tumor with extradural metastases. Cancer 26:239-244, 1970

22. Willis AG, Birrell JH: The structure of a carotid body tumor. Acta Anat (Basel) 25:220-265, 1955

\section{Disclosures}

The authors report no conflict of interest concerning the materials or methods used in this study or the findings specified in this paper.

\section{Author Contributions}

Conception and design: Jeevan, Saleh. Drafting the article: Jeevan, Saleh, LaBagnara. Critically revising the article: Jeevan, Neil, Hillard. Reviewed submitted version of manuscript: Jeevan.

\section{Correspondence}

Dhruve S. Jeevan, Department of Neurosurgery, New York Medical College, Munger Pavilion, Rm. 329, Valhalla, NY 10595. email: dhruve.jeevan@me.com. 